\title{
Significance of positive Stenotrophomonas maltophilia culture in acute respiratory tract infection
}

\section{A. Pathmanathan and G.W. Waterer}

ABSTRACT: Stenotrophomonas maltophilia is a common coloniser of the respiratory tract of patients with chronic lung disease, and, in the absence of pneumonia or bacteraemia, is often ignored by physicians at the Royal Perth Hospital (Perth, Australia). Experience at the Royal Perth Hospital was reviewed to determine whether ignoring $S$. maltophilia in this setting has any apparent effect on clinical outcome.

All patients who presented with an acute respiratory illness and yielded a positive culture for $\mathbf{S}$. maltophilia between 1995 and 2002 were retrospectively reviewed. All subjects had to yield a positive respiratory isolate of $S$. maltophilia and undergo chest radiography within $24 \mathrm{~h}$ of the isolate being obtained.

Ninety-two episodes were identified in 89 individuals; 64 showed no evidence of consolidation. Of the study group, $51(80.0 \%)$ received no anti-S. maltophilia antibiotic therapy and $21(32.8 \%)$ had a nosocomially acquired isolate. The overall mortality rate was $20.3 \%$. There was no impact of anti-S. maltophilia therapy on outcome. The only independent predictor of mortality was serum albumin level.

As there was no measurable impact of antibiotic therapy, in the absence of consolidation, a positive respiratory tract isolate of Stenotrophomonas maltophilia probably represents colonisation of a severely impaired host rather than invasive disease.

\section{KEYWORDS: Outcome, respiratory, Stenotrophomonas maltophilia, treatment}

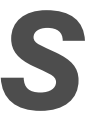
tenotrophomonas maltophilia is a Gramnegative nonfermentive bacillus. It has previously been designated as Pseudomonas maltophilia and Xanthomonas maltophilia [1]. Owing to a combination of high innate antibiotic resistance, including two chromosomal cephalosporinases, one of which hydrolyses carbapenems [2], and selective antibiotic pressure, S. maltophilia is emerging as an important nosocomial pathogen.

Mortality rates of $10-60 \%$ in patients with bacteraemia due to $S$. maltophilia have been reported [2-5], largely influenced by their occurrence in critically ill, heavily immunosuppressed patients. However, the attributable mortality due to $S$. maltophilia bacteraemia appears to be equivalent to that for other nosocomial bacteraemias after adjusting for underlying disease status [6].

Although the respiratory system is the most common site of isolation and infection with S. maltophilia [7], the significance of a positive respiratory tract isolate in the absence of bacteraemia is less clear as transient asymptomatic carriage is not common, especially in the nosocomial setting [8-10]. Making a distinction between S. maltophilia colonisation and infection is made even more difficult by the frequent isolation of other organisms from the same specimen [8].

Therefore, although there is good evidence that $S$. maltophilia causes significant mortality in patients with nosocomial pneumonia [8, 11], in other clinical settings, the significance of a positive respiratory isolate is much less clear.

At the Royal Perth Hospital (Perth, Australia), there was quite a range of opinions regarding the need to treat $S$. maltophilia when isolated from sputum in the absence of pneumonia. Therefore, all case records from the period of 1995-2002 were reviewed to determine whether the decision to treat S. maltophilia impacted on outcome, and whether clinical indications for treatment could be determined.

\section{METHODS}

Patient identification and selection

All patients yielding a respiratory tract isolate of S. maltophilia at the Royal Perth Hospital between

\section{AFFILIATIONS}

Dept of Respiratory Medicine, Royal Perth Hospital, Perth, Australia.

CORRESPONDENCE

G.W. Waterer

School of Medicine and Pharmacology University of Western Australia Dept of Respiratory Medicine Royal Perth Hospital GPO Box X2213 Perth 6847

Western Australia Australia

Fax: 61892240246

E-mail: waterer@cyllene.uwa.edu.au

Received:

August 182004

Accepted after revision:

December 302004 
1995 and 2002 were identified from the hospital microbiology database. In order to qualify for this analysis, patients had to be documented as having at least two of the following at the time of the sputum culture: 1) increased dyspnoea; 2) productive cough; 3) fever; and 4) deterioration in oxygenation. Patients had to be hospitalised or in the emergency department at the time the respiratory sample was obtained to be included in the study. Case records were then reviewed for the data points of interest. Patients with radiological evidence of pneumonia were excluded from analysis of impact on therapy. This study was approved by the Ethics Committee of the Royal Perth Hospital.

\section{Definitions}

An episode of $S$. maltophilia was defined as a positive sputum sample, endotracheal tube aspirate, bronchial wash or lavage culture. If no specific therapy for S. maltophilia was given, then subsequent isolates obtained during the same hospital admission were not defined as a separate episode.

Nosocomial infection was defined as a positive culture for S. maltophilia $>72 \mathrm{~h}$ after admission to hospital, or a positive culture on admission if the patient had been discharged from hospital in the past 14 days.

With respect to outcome, mortality was defined as death occurring within 14 days of the initial positive culture of S. maltophilia.

Applied Physiology, Age, and Chronic Health Evaluation (APACHE) II scores [12] were calculated from the worst physiological values on the day that the respiratory isolate was obtained.

\section{Statistics}

Differences in continuous variables were assessed using an unpaired t-test after checking for normality of distribution. Differences in categorical variables were assessed with Fisher's exact test. A p-value of $<0.05$ was considered significant.

\section{RESULTS}

A total of 89 individuals were identified as yielding a positive respiratory tract culture for $S$. maltophilia on 92 separate occasions. The mean \pm SD age of the patients was $60.2 \pm$ 18.1 yrs (range 19-95 yrs). There were 35 (39.3\%) female and $54(60.7 \%)$ male patients. The clinical diagnoses at the time of sputum culture were acute tracheobronchitis in $30(32 \%)$ patients, acute exacerbation of chronic obstructive pulmonary disease in 27 (29\%), nosocomial pneumonia in $17(18 \%)$, community-acquired pneumonia in $11(12 \%)$ and infective exacerbation of bronchiectasis in seven $(7.5 \%)$. Of the nosocomial pneumonias, 15 out of 17 were in patients requiring mechanical ventilation (i.e. with suspected ventilator-associated pneumonia).

In $48(52.2 \%)$ episodes, S. maltophilia was not the only respiratory pathogen cultured from the positive sample, with three or more potential pathogens being identified on eight $(8.7 \%)$ occasions. The most common copathogens isolated were P. aeruginosa $(\mathrm{n}=16)$, Klebsiella pneumoniae $(\mathrm{n}=5)$, Enterobacter spp. $(n=6)$, Staphylococcus aureus $(n=4)$, Escherichia coli $(n=3)$ and Acinetobacter spp. $(n=3)$. Copathogens were identified in $14(36.8 \%)$ of the 38 nosocomially acquired episodes and 20
$(37.0 \%)$ of the community-acquired episodes ( $p>0.1)$. Amongst survivors, an additional sputum sample was obtained 728 days after the initial culture in $36 \%$ of those who did not receive specific anti-S. maltophilia antibiotic therapy and $57 \%$ of those who did. There was no documented chronic carriage of S. maltophilia in the treated or untreated groups.

Table 1 shows a comparison of the episodes with and without radiological evidence of pneumonia. There were no significant differences between episodes with and without pneumonia for the variables shown in table 1 . The most common antibiotics received in the 30 days prior to the first positive culture for $S$. maltophilia were third-generation cephalosporins $(n=27)$, a carbapenem $(n=10)$, a macrolide $(n=7)$ and a $\beta$-lactam $/ \beta$ lactamase inhibitor combination $(n=6)$. All third-generation cephalosporins and carbapenems were received within the 10 days prior to the positive culture for S. maltophilia. Apart from the four patients who had a second identified episode, only one other patient had a previously documented infection with S. maltophilia. With respect to other prior culture of multidrug-resistant pathogens, eight patients had documentation of previous culture of $P$. aeruginosa, two of whom had documented bronchiectasis. No patient had cystic fibrosis. With respect to malignancies, 15 were solid-organ malignant tumours and eight haematological malignancies.

As the vast majority (82\%) of patients with pneumonia received anti-S. maltophilia antibiotics and significant mortality has been attributed to pneumonia due to this organism [8, 11], further analysis was limited to the patients without radiological evidence of pneumonia. Table 2 shows a summary of the demographic information for both community-acquired and nosocomially acquired episodes after excluding the $28(30.4 \%)$

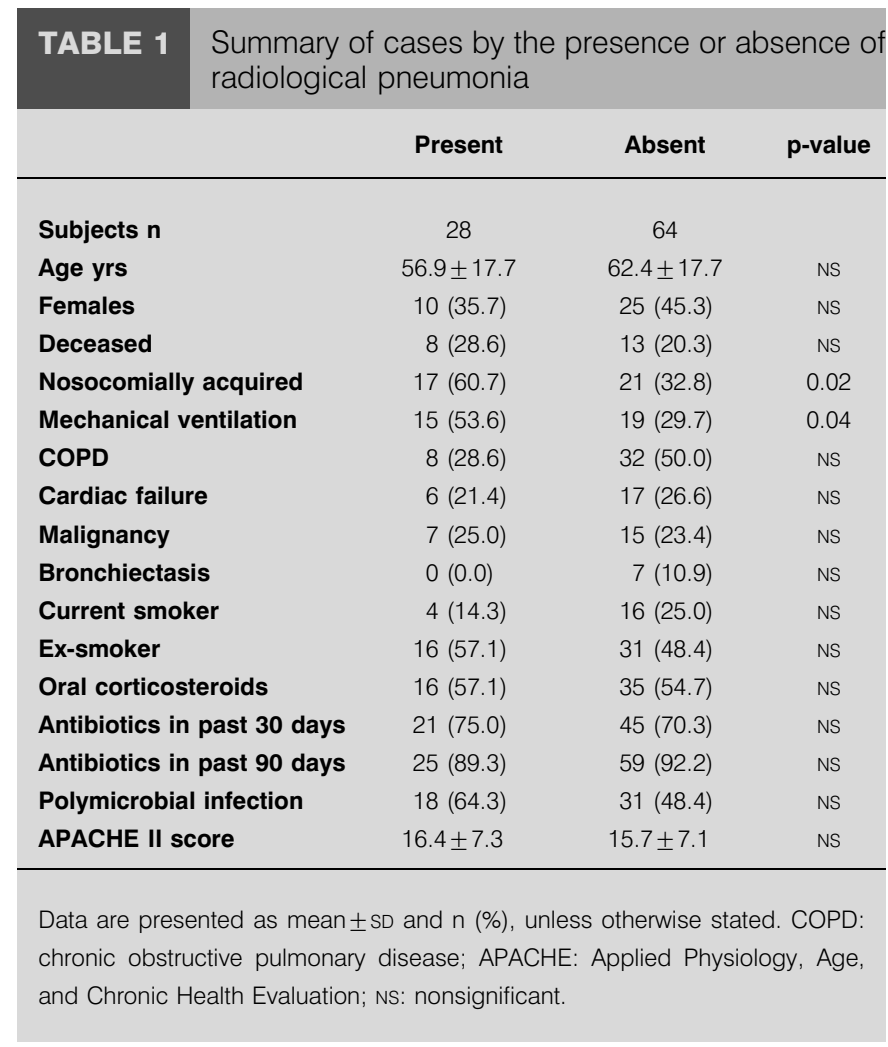




\begin{tabular}{|c|c|c|c|c|}
\hline \multirow[t]{2}{*}{ TABLE 2} & \multicolumn{4}{|c|}{$\begin{array}{l}\text { Demographic information for community-acquirec } \\
\text { and nosocomially acquired Stenotrophomonas } \\
\text { maltophilia isolates from patients without } \\
\text { pneumonia }\end{array}$} \\
\hline & & Community & Nosocomial & p-value \\
\hline \multicolumn{2}{|l|}{ Subjects $n$} & 43 & 21 & \\
\hline \multicolumn{2}{|l|}{ Age yrs } & $64.9 \pm 18.2$ & $57.3 \pm 16.0$ & NS \\
\hline \multicolumn{2}{|l|}{ Females } & $15(34.9)$ & $14(66.7)$ & 0.03 \\
\hline \multicolumn{2}{|l|}{ Deceased } & $5(11.6)$ & $8(38.1)$ & 0.02 \\
\hline \multicolumn{2}{|c|}{ Anti-S. maltophilia therapy } & $15(34.9)$ & $3(14.3)$ & NS \\
\hline \multicolumn{2}{|c|}{ Deceased } & 5 (11.6) & $8(38.1)$ & NS \\
\hline \multicolumn{2}{|c|}{ Mechanical ventilation } & $4(9.3)$ & $17(80.1)$ & $<0.0001$ \\
\hline \multicolumn{2}{|c|}{ COPD } & $24(55.8)$ & 7 (33.3) & NS \\
\hline \multicolumn{2}{|c|}{ Cardiac failure } & $12(27.9)$ & $5(23.8)$ & NS \\
\hline \multicolumn{2}{|c|}{ Malignancy } & $10(23.2)$ & $5(23.8)$ & NS \\
\hline \multicolumn{2}{|c|}{ Bronchiectasis } & 7 (16.3) & $0(0.0)$ & NS \\
\hline \multicolumn{2}{|c|}{ Current smoker } & $8(18.6)$ & $8(38.1)$ & NS \\
\hline \multicolumn{2}{|c|}{ Ex-smoker } & $25(58.1)$ & $6(28.6)$ & NS \\
\hline \multicolumn{2}{|c|}{ Oral corticosteroids } & $29(67.4)$ & $6(28.6)$ & 0.007 \\
\hline \multicolumn{2}{|c|}{ Antibiotics in past 30 days } & $24(55.8)$ & $21(100)$ & 0.0001 \\
\hline \multicolumn{2}{|c|}{ Antibiotics in past 90 days } & $38(88.4)$ & $21(100)$ & NS \\
\hline \multicolumn{2}{|c|}{ Polymicrobial infection } & $17(39.5)$ & $14(66.7)$ & 0.04 \\
\hline
\end{tabular}

episodes with pneumonia. The only significant differences were the expected higher frequency of mechanical ventilation in patients with nosocomially acquired infection $(\mathrm{p}<0.0001)$, the greater frequency of antibiotic use in the past 30 days $(p=0.0001)$, greater use of corticosteroids $(p=0.007)$ and the greater frequency of polymicrobial infection $(p=0.04)$ in the same group. Anti-S. maltophilia therapy was sulfamethoxazole/trimethoprim with $(n=6)$ or without $(n=12)$ ticarcillin/ clavulanic acid.

Table 3 compares the fatal and nonfatal cases with respect to key comorbid and clinical features. Compared with survivors, nonsurvivors exhibited a substantially lower white cell count at the time of culture $(p=0.02)$ and a substantially higher serum albumin concentration $(\mathrm{p}<0.0001)$, and were significantly more likely to have additional pathogens identified in the same respiratory sample $(p=0.005)$. The difference in maximum recorded respiratory frequency also approached significance $(p=0.06)$. As would be expected, the APACHE II score was also substantially lower in survivors $(p<0.0001)$.

Logistic regression analysis incorporating all of the factors in table 3 (except composite APACHE II score) plus other chronic organ failures and significant interactions found that the only independent predictor of death was serum albumin concentration $(p=0.006)$. Neither anti-S. maltophilia therapy $(p=0.485)$ nor polymicrobial infection $(\mathrm{p}=0.124)$ was an independent predictor of outcome.

Analysis of patients who did and did not receive specific antiS. maltophilia therapy was conducted at both a univariate (table 4) and multivariate level, incorporating all of the factors in tables 2 and 3. No comorbid or clinical feature, including

\section{TABLE 3 Summary of fatal and nonfatal cases without pneumonia}

\begin{tabular}{|c|c|c|c|}
\hline & Fatal & Nonfatal & $p$-value \\
\hline Subjects $\mathbf{n}$ & 13 & 51 & \\
\hline Nosocomially acquired & $8(61.6)$ & $13(25.5)$ & 0.02 \\
\hline Anti-S. maltophilia therapy & $4(30.8)$ & $14(27.5)$ & NS \\
\hline Age yrs & $59.2 \pm 16.6$ & $63.1 \pm 18.1$ & NS \\
\hline Females & $6(46.2)$ & $23(45.1)$ & NS \\
\hline Mechanical ventilation & $8(61.6)$ & $11(21.6)$ & 0.01 \\
\hline COPD & $4(30.8)$ & 27 (52.9) & NS \\
\hline Cardiac failure & $1(7.7)$ & $16(31.4)$ & NS \\
\hline Malignancy & $3(23.1)$ & $12(23.5)$ & NS \\
\hline Maximum $\mathrm{fc}$ beats $\cdot \mathrm{min}^{-1}$ & $113.9 \pm 22.2$ & $105.9 \pm 19.1$ & NS \\
\hline Maximum $f R$ breaths $\cdot \min ^{-1}$ & $27.4 \pm 7.4$ & $24.2 \pm 4.6$ & NS \\
\hline White cell count $10^{9}$ cells $\cdot \mathrm{L}^{-1}$ & $14.9 \pm 7.1$ & $10.2 \pm 5.9$ & 0.02 \\
\hline Serum albumin $\mathbf{g} \cdot \mathrm{L}^{-1}$ & $24.3 \pm 5.3$ & $32.9 \pm 6.7$ & $<0.0001$ \\
\hline Polymicrobial infection & $11(84.6)$ & 20 (39.2) & 0.005 \\
\hline APACHE II score & $19.3 \pm 9.4$ & $10.4 \pm 5.9$ & $<0.0001$ \\
\hline
\end{tabular}

Data are presented as mean \pm SD and $n(\%)$, unless otherwise stated. COPD: chronic obstructive pulmonary disease; $f \mathrm{C}$ : cardiac frequency; fR: respiratory frequency; APACHE: Applied Physiology, Age, and Chronic Health Evaluation NS: nonsignificant.

APACHE II score, was found that predicted selection of specific anti-S. maltophilia therapy. The apparent trend to lower white cell count in those treated was not significant $(p=0.09)$.

The various subsets were also analysed for an influence of specific anti-S. maltophilia therapy on nonmortality outcomes. For community-acquired isolates, there was no significant

TABLE 4 Comparison of treated and untreated episodes

\begin{tabular}{|c|c|c|c|}
\hline & Treated & Untreated & p-value \\
\hline Subjects $n$ & 18 & 51 & \\
\hline Nosocomially acquired & $2(11.1)$ & 19 (37.3) & NS \\
\hline Females & $6(33.3)$ & $23(45.1)$ & NS \\
\hline Age yrs & $58.7 \pm 19.7$ & $63.7 \pm 17.0$ & NS \\
\hline COPD & $9(50.0)$ & $22(43.1)$ & NS \\
\hline Mechanical ventilation & $2(11.1)$ & 17 (33.3) & NS \\
\hline Cardiac failure & $3(16.7)$ & $14(27.5)$ & NS \\
\hline Malignancy & $5(27.8)$ & $10(19.6)$ & NS \\
\hline Maximum fc beats $\cdot \mathrm{min}^{-1}$ & $105.9 \pm 20.9$ & $108.4 \pm 19.7$ & NS \\
\hline Maximum $f_{R}$ breaths $\cdot \mathrm{min}^{-1}$ & $22.6 \pm 3.7$ & $25.8 \pm 5.8$ & NS \\
\hline White cell count $10^{9}$ cells $\cdot L^{-1}$ & $8.8 \pm 6.4$ & $12.0 \pm 6.2$ & NS \\
\hline Serum albumin $\mathbf{g} \cdot \mathrm{L}^{-1}$ & $30.8 \pm 6.9$ & $31.2 \pm 7.5$ & NS \\
\hline Polymicrobial infection & $9(50.0)$ & $22(43.1)$ & NS \\
\hline APACHE II score & $15.9 \pm 6.9$ & $16.1 \pm 7.3$ & NS \\
\hline
\end{tabular}

Data are presented as mean \pm SD and $n(\%)$, unless otherwise stated. COPD chronic obstructive pulmonary disease; $f \mathrm{C}$ : cardiac frequency; $f_{\mathrm{R}}$ : respiratory frequency; APACHE: Applied Physiology, Age, and Chronic Health Evaluation; NS: nonsignificant. 
difference in the length of hospital stay in survivors who received specific anti-S. maltophilia therapy (mean \pm SD $20.2 \pm$ 14.5 days) compared with those who did not (16.4 \pm 15.4 days). Similarly, there was no difference in the proportion of patients readmitted with a respiratory tract infection in the 3 months after discharge from hospital (therapy group $27 \%$; nontherapy group $26 \%$ ).

\section{DISCUSSION}

Although nosocomial bacteraemia and ventilator-associated pneumonia due to $S$. maltophilia show significant mortality and morbidity $[8,11]$, it was not possible to attribute any excess mortality or morbidity in the absence of pneumonia. Although overall mortality rates were high, the absence of any apparent effect of treatment is more consistent with the acquisition of $S$. maltophilia being a marker of severe underlying life-limiting illnesses than of $S$. maltophilia being a highly virulent pathogen in this setting. Given the high frequency of multiple pathogens, in the absence of consolidation, isolation of S. maltophilia may not require antibiotic therapy as the majority of patients in this group do not appear to benefit from treatment.

The argument that most isolates of $S$. maltophilia from the respiratory tract represent colonisation rather than invasive disease is supported by several findings. First, and most importantly, specific anti-S. maltophilia antibiotic therapy did not alter the outcome in patients without pneumonia. Secondly, even without antibiotic therapy, the overwhelming majority of patients cleared S. maltophilia from their respiratory tract. Thirdly, in the majority of cases, S. maltophilia was not the only pathogen isolated. Finally, the only independent predictor of survival was serum albumin concentration. This strongly suggests that the isolation of $S$. maltophilia is an indication of a severely compromised host rather than S. maltophilia being an extremely virulent opportunistic pathogen.

Although treatment of $S$. maltophilia may not be critical, physicians still need to be cautious in their selection of antibiotic therapy when it is isolated. Given the extremely high frequency of multiple pathogens observed in the present study and in others $[7,8,10]$, limiting antibiotic therapy to cover only $S$. maltophilia may be dangerous, as many of the typical copathogens are resistant to sulfamethoxazole/ trimpethoprim. It should also be noted that both gatifloxacin and doxycycline appear to be good alternatives to sulfamethoxazole/trimethoprim in vitro [13], although efficacy in clinical trials has not been assessed.

Given the retrospective observational nature of the present study, there are clearly limitations to the interpretation of the data. In particular, the possibility that physicians selected patients for treatment based on an unidentifiable criterion cannot be excluded. However, the comparison shown in table 4 suggests that the treated and untreated groups were very similar. Furthermore, the finding that the only independent predictor of mortality was serum albumin concentration strengthens the argument that a respiratory isolate of $S$. maltophilia indicates a severely compromised host rather than invasive disease with a highly virulent pathogen.

In summary, in the absence of pneumonia-specific antibiotic therapy, Stenotrophomonas maltophilia isolated from the respiratory tract does not appear to affect outcome. When
Stenotrophomonas maltophilia is isolated, physicians should be alert to the high probability of multiple pathogens being present. Although respiratory tract colonisation does not appear to have adverse implications, the isolation of Stenotrophomonas maltophilia indicates a severely compromised host with a high likelihood of mortality attributable to the underlying disease processes.

\section{REFERENCES}

1 Palleroni NJ, Bradbury JF. Stenotrophomonas, a new bacterial genus for Xanthomonas maltophilia (Hugh 1980) Swings et al. 1983. Int J Syst Bacteriol 1993; 43: 606-609.

2 Spencer RC. The emergence of epidemic, multipleantibiotic-resistant Stenotrophomonas (Xanthomonas) maltophilia and Burkholderia (Pseudomonas) cepacia. J Hosp Infect 1995; 30: Suppl. 1, 453-464.

3 Muder RR, Harris AP, Muller S, et al. Bacteremia due to Stenotrophomonas (Xanthomonas) maltophilia: a prospective, multicenter study of 91 episodes. Clin Infect Dis 1996; 22: 508-512.

4 Morrison AJ, Hoffman KK, Wenzel RP. Associated mortality and clinical characteristics of nosocomial Pseudomonas maltophilia in a university hospital. J Clin Microbiol 1986; 24: 52-55.

5 Fiedman ND, Korman TM, Fairley CK, et al. Bacteraemia due to Stenotrophomonas maltophilia: an analysis of 45 episodes. J Infect 2002; 45: 47-53.

6 Senol E, DesJardin J, Stark PC, et al. Attributable mortality of Stenotrophomonas maltophilia bacteremia. Clin Infect Dis 2002; 34: 1653-1656.

7 Gales AC, Jones RN, Forward KR, Linares J, Sader HS, Verhoef J. Emerging importance of multidrug-resistant Acinetobacter species and Stenotrophomonas maltophilia as pathogens in seriously ill patients: geographic patterns, epidemiological features, and trends in SENTRY antimicrobial surveillance program (1997-1999). Clin Infect Dis 2001; 32: Suppl. 2, S104-S113.

8 Gopalakrishnan R, Hawley HB, Czachor JS, Markert RB, Bernstein JM. Stenotrophomonas maltophilia infection and colonization in the intensive care units of two community hospitals: a study of 143 patients. Heart Lung 1999; 28: 134-141.

9 Valdezate S, Vinel A, Martin-Davila P, et al. High genetic diversity among Stenotrophomonas maltophilia strains despite their originating at a single hospital. J Clin Microbiol 2004; 42: 693-699.

10 del Toro MD, Rodriguez-Bano J, Herrero M, et al. Clinical epidemiology of Stenotrophomonas maltophilia colonization and infection: a multicenter study. Medicine (Baltimore) 2004; 81: 228-239.

11 Trouillet J-L, Chastre J, Vuagnat A, et al. Ventilatorassociated pneumonia caused by potentially drug-resistant bacteria. Am J Respir Crit Care Med 1998; 157: 531-539.

12 Knaus WA, Draper EA, Wagner DP, et al. APACHE II: a severity of disease classification system. Crit Care Med 1985; 13: 818-829.

13 Nicodemo AC, Araujo MR, Ruiz AS, Gales AC. In vitro susceptibility of Stenotrophomonas maltophilia isolates: comparison of disc diffusion, Etest and agar dilution methods. J Antimicrob Chemother 2004; 53: 604-608. 